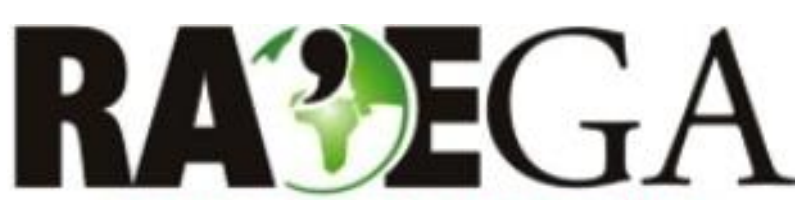

O ESPAÇO GEOGRÁFICO EM ANÁLISE

\title{
VERIFICAÇÃO DA QUALIDADE DE ORTOMOSAICOS PRODUZIDOS A PARTIR DE IMAGENS OBTIDAS COM AERONAVE REMOTAMENTE PILOTADA SEM O USO DE PONTOS DE APOIO
}

\section{QUALITY ASSESSMENT IN ORTHOMOSAICS PRODUCED FROM IMAGES OBTAINED WITH UNMANNED AERIAL VEHICLE WITHOUT THE USE OF SUPPORT POINTS}

\author{
Carlos Henrique Sopchaki ${ }^{1}$, Otacílio Lopes de Souza da Paz ${ }^{2}$, Niarkios Luiz Santos de Salles Graça ${ }^{3}$, Tony \\ Vinicius Moreira Sampaio ${ }^{4}$
}

\section{RESUMO}

As RPAs (Aeronaves Remotamente Pilotadas) têm sido amplamente utilizadas na geração de ortomosaicos e modelos digitais de elevação (MDE), que subsidiam as análises geoespaciais. Por vezes, estas análises demandam levantamentos rápidos e/ou são feitos em áreas remotas, o que inviabiliza o uso de RPAs associadas a pontos de apoio para a correção da posição, ajuste e avaliação da qualidade dos ortomosaicos e MDEs. Atualmente é possível a geração automatizada destes produtos através do uso de rotinas preestabelecidas disponíveis em programas especializados na manipulação das imagens obtidas pelas RPAs. Este trabalho avaliou a qualidade planimétrica dos ortomosaicos produzidos a partir de imagens coletadas com RPAs criados a partir do uso de rotinas automatizadas de software especialista, sem o emprego de pontos de apoio de campo. Como resultado observou-se que $100 \%$ dos ortomosaicos produzidos enquadram-se na Classe A da escala 1:50.000 ou superior (PEC-PCD - Padrão de Exatidão Cartográfica dos Produtos Cartográficos Digitais), sendo a melhor acurácia planimétrica obtida compatível com a escala 1:5.000. Os ortomosaicos apresentaram padrão regular de distribuição do erro, não apresentando áreas preferenciais de deformação. Os erros nas distâncias obtidas nos ortomosaicos são inferiores a 1,6\% ao valor da distância medida..

Palavras-chave: Acurácia; Adequação ao uso; Análise geoespacial; Drone; RPA.

\section{ABSTRACT}

UAVs (Unmanned aerial vehicle) have been widely used in the generation of orthomosaics and digital elevation models (DEM) that give support geospatial analyzes. Sometimes these analyzes require rapid surveys and/or are make in remote areas, which makes it unfeasible to use UAVs associated with the points of support for the correction of position, adjustment and evaluation of the quality of orthomosaic and MDEs. Currently it is possible to automate the generation of these products using pre-established routines available in specialized programs to manipulation UAV's images. This work evaluates the planimetric quality of the orthomosaics produced from images collected with UAVs associated with the use of automated routines of specialist software, without the use of support points. As a result, it was observed that $100 \%$ of the orthomosaics obtained was classified into Class A of the scale 1: 50,000 or higher (PEC-PCD - Cartographic Accuracy Standard of Digital Cartographic Products) and, the best planimetric accuracy was 1:5000. The orthomosaics showed regular pattern of error distribution, not presenting preferred areas of deformation (edge / center). The errors in the measured distances in orthomosaics are less than $1.6 \%$ of the measured distance.

Key-words: Accuracy; Fitness to use; Geospatial analysis; RPA; UAV.

\footnotetext{
1 Universidade Federal do Paraná, Curitiba/PR, e-mail: csopchaki@gmail.com

2 Universidade Federal do Paraná, Curitiba/PR, e-mail: otacilio.paz@gmail.com

${ }^{3}$ Universidade Federal do Paraná, Curitiba/PR, e-mail: niarkiosnl@hotmail.com

${ }^{4}$ Universidade Federal do Paraná, Curitiba/PR, e-mail: tonysampaio@ufpr.br
} 


\section{VERIFICAÇÃO DA QUALIDADE DE ORTOMOSAICOS PRODUZIDOS A PARTIR DE IMAGENS OBTIDAS COM AERONAVE REMOTAMENTE PILOTADA SEM O USO DE PONTOS DE APOIO}

\section{INTRODUÇ̃̃o}

Aeronaves Remotamente Pilotadas, conhecidas também como Veículo Aéreo NãoTripulado (VANT), UAV em inglês (Unmanned aerial vehicle) ou, popularmente, "Drones" no Brasil, têm permitido a obtenção de dados para estudos e pesquisas em diferentes áreas (HARDIN; JENSEN, 2011). RPA é termo adotado pela Agência Nacional de Aviação Civil (ANAC, 2015) para designar os VANTs e resulta da junção das iniciais do termo Remotely Piloted aircraft RPA (EVERAERTS, 2008).

Existem diferenças conceituais e técnicas entre as RPAs, sendo a principal delas o sistema aerodinâmico, que divide as mesmas em asa fixa e multirotor (SOUSA; SOUSA; COSTA, 2016). Diferentes classificações ainda podem ser utilizadas quanto ao peso e uso das mesmas (DECEA, 2017).

Quanto ao sistema de navegação embarcado, dois tipos são encontrados no mercado: RPAs com RTK (Real Time Kinematic) e sem RTK, denominadas RPA-SPS (Standard Positioning Service). Enquanto o primeiro permite a correção da posição em tempo real e o processamento das posições observadas, o segundo realiza a navegação utilizando somente os sinais abertos de uma ou mais constelações de satélites de navegação (GPS, GLONASS, Galileu, etc.) e não possibilita a correção e o reprocessamento das coordenadas (DEPARTMENT OF DEFENSE, 2008).

Dos produtos que podem ser obtidos com o uso de RPAs, dois se destacam nas análises geoespaciais: o ortomosaico e o modelo digital de elevação (MDE). O ortomosaico corresponde ao mosaico das ortofotos (MARCHETTI; GARCIA, 1989; TORRADO, 2016) e, o MDE, a imagem na qual cada pixel apresenta um valor relativo à altitude da superfície modelada (SOPCHAKI, 2016).

Estes materiais servem de base para obtenção de medidas planimétricas e altimétricas que subsidiam as análises geoespaciais. Assim, são utilizados na delimitação de áreas de risco (SOLOMON et al., 2002), quantificação de cicatrizes de movimentos de massa (LUCIEER;
JONG; TURNER, 2014), definição de áreas de proteção permanente (APP) (CÂNDIDO; DA SILVA; FILHO, 2015), classificação e quantificação da vegetação (PRIMICERIO et al., 2012; BRAZ et al., 2015), delimitação e quantificação de áreas de inundação (SUMALAN; POPESCU; ICHIM, 2015; BUFFON; PAZ; SAMPAIO, 2017), etc.

A partir de 2015, houve um aumento no número de pesquisas que utilizam dados obtidos com uso de RPAs (LONGHITANO, 2010; SOUSA, 2017). Isto se deu em função de diferentes fatores como: facilidade de uso, resolução das imagens, custo com aquisição do equipamento e processamento das imagens e, a possibilidade de adequar a data da coleta e repetição dos levantamentos à dinâmica dos eventos pesquisados.

A facilidade de uso pode ser caracterizada a partir de dois aspectos:

1. planejamento dos voos: podem ser feitos previamente em escritório ou diretamente em campo utilizando smartphones (ou tablets) com uso de softwares gratuitos (ex.: Pix4D Capture, Precision Hawk, DroneDeploy, etc.). Tratase de um procedimento em três etapas: desenho do polígono (ou linha ou circunferência) que recobre a área de interesse, definição dos recobrimentos do voo (lateral e longitudinal) e, da altura do voo (responsável pela resolução espacial final dos produtos). Cabe observar que nestes casos, da decolagem ao pouso o controle da aeronave é feito pelo aplicativo que gerencia o plano de voo e a RPA.

2. processamento das imagens: os produtos finais podem ser processados via web ou diretamente pelo usuário. No processamento pela web, o usuário envia as imagens capturadas e recebe os produtos possíveis de serem elaborados com as mesmas (MDT, MDS, ortomosaico, índice de vegetação, etc.). No processamento desktop, o usuário pode utilizar programas de Sistemas de Informações Geográficas - SIG/ 


\section{VERIFICAÇÃO DA QUALIDADE DE ORTOMOSAICOS PRODUZIDOS A PARTIR DE IMAGENS OBTIDAS COM AERONAVE REMOTAMENTE PILOTADA SEM O USO DE PONTOS DE APOIO}

Processamento Digital de Imagens - PDI (como: ArcGIS, ENVI, QGIS, SPRING, etc.), ou programas especializados na manipulação deste tipo de dado (como a exemplo: Agisoft Photoscan, Pix4Dmapper e Correlator3D). Estes últimos acessam diretamente os metadados das imagens e, através de rotinas preestabelecidas, ou por manipulação do usuário, realizam a geração dos produtos.

$O$ custo pode ser dividido em custo com equipamentos e custo final dos produtos gerados. O custo dos produtos possui relação com o tipo e a qualidade que deseja e, com a dimensão da área a ser mapeada. Neste caso são considerados os gastos com deslocamento, aquisição de pontos de controle, aluguel ou compra de equipamentos e softwares, contratação de mão-de-obra, etc. O custo dos equipamentos depende, dentre outros, do sistema de posicionamento da RPA (RTK ou SPS) e do tipo, quantidade e qualidade dos sensores embarcados.

Os trabalhos de Perin et al. (2016) e Zanetti (2017) demonstram que, com auxílio de pontos de apoio (pontos com coordenadas conhecidas), mesmo RPAs-SPS com sistema fotográfico de uso recreativo, podem ser empregados na geração de ortomosaicos e MDEs permitindo a obtenção de produtos com escala de 1:1000 ou melhores.

O nível de simplificação operacional, que tem permitido a usuários sem conhecimento técnico na área de mapeamento gerar ortomosaicos e MDEs, tem explicação na ampla gama de aplicação das RPAs, o que tem levado as empresas que comercializam produtos, principalmente, para agricultura, mineração e arquitetura, a desenvolver e aprimorar tais rotinas (NEX; REMONDINO, 2014).

Apesar de conhecidos os padrões de acurácia das coordenadas obtidas com o uso dos equipamentos de navegação embarcados nas RPAs-SPS (GPS, GLONASS, etc.), ainda não são perfeitamente conhecidos os reflexos da propagação e acumulação de erros sobre os produtos cartográficos gerados com o uso destes equipamentos (ortomosaico e MDE), sem o uso de pontos de apoio.

Considerando que o levantamento de dados em campo por vezes demanda a realização de trabalhos rápidos (como o imageamento de um movimento de massa) e/ou a obtenção de dados em áreas de difícil acesso (sendo inviável a aquisição de pontos de apoio e controle) e, principalmente, considerando o custo e a facilidade de geração de ortomosaicos e MDEs com RPAs-SPS, cabe verificar qual seria a qualidade posicional esperada para os produtos cartográficos gerados empregando somente estes equipamentos associados a rotinas padronizadas de programas especializados.

O conhecimento dos padrões de acurácia esperados permitiria aos pesquisadores e técnicos que demandam estes produtos (ortomosaico e MDE) decidir sobre a adequação e pertinência de uso destes equipamentos e produtos em suas pesquisas e análises geoespaciais.

No que se refere à qualidade planimétrica, dois tipos de erros vão estar presentes nos ortomosaicos (DA SILVA et al., 2015): 1. O deslocamento em relação a sua posição esperada no espaço (qualidade absoluta erro que interfere diretamente no enquadramento do produto cartográfico em relação à escala (PEC-PCD) (DSG - DIRETORIA DO SERVIÇO GEOGRÁFICO, 2016b) e, 2. Deslocamentos internos a imagem (qualidade relativa) que irão afetar diretamente as métricas obtidas a partir destes produtos.

Com base nos padrões estabelecidos pelo Padrão de Exatidão Cartográfica dos Produtos Cartográficos Digitais (PEC-PCD) objetiva-se avaliar a qualidade planimétrica dos ortomosaicos produzidos a partir de imagens coletadas com RPAs-SPS e uso de rotinas automatizadas de software especialista, sem o emprego de pontos de apoio.

\section{MATERIAIS E MÉTODOS}

A pesquisa foi dividida em quatro fases: 1) planejamento dos voos; 2) realização dos levantamentos; 3) processamentos 


\section{VERIFICAÇÃO DA QUALIDADE DE ORTOMOSAICOS PRODUZIDOS A PARTIR DE IMAGENS OBTIDAS COM AERONAVE REMOTAMENTE PILOTADA SEM O USO DE PONTOS DE APOIO}

dos dados e geração dos ortomosaicos e 4) análise de qualidade planimétrica. Foi utilizada uma RPA modelo Phantom 3 - Professional, cedida pelo Laboratório de Análises de Padrões Espaciais e Cartografia Temática (LAPE-CT) da Universidade Federal do Paraná (UFPR).

Este modelo integra dados inerciais e dados GNSS para sua navegação e determinação da posição de cada imagem no instante da aquisição. As informações de posição são gravadas instantaneamente nos metadados de cada imagem (GUJJAR et al., 2017;
MOHAMMAD et al., 2017). Maiores detalhes sobre o equipamento podem ser obtidos junto ao fabricante (DJI, 2017).

Os levantamentos foram realizados no Centro Politécnico, Campus III da Universidade Federal do Paraná (figura 1). A área de voo foi definida em 42,64 ha, em cinco faixas de voo, com recobrimento lateral de $70 \%$ e longitudinal de $80 \%$ e velocidade de $10 \mathrm{~m} / \mathrm{s}$. Foi utilizado o aplicativo livre Pix4D Capture para o planejamento dos voos.

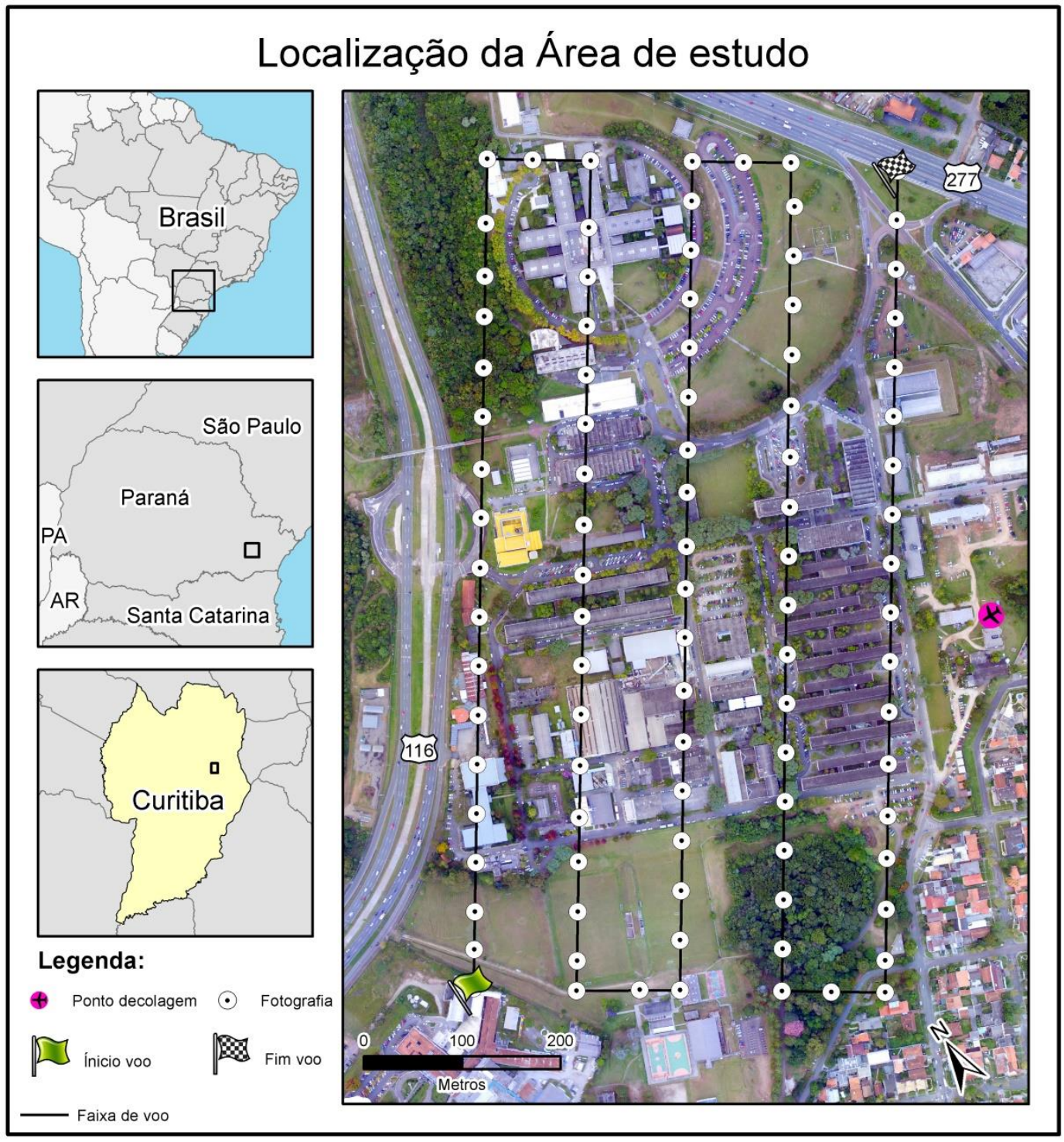

Figura 1 - Localização do Centro Politécnico da UFPR (área de estudo) e faixas de voo da RPA. Fonte: autores (2017).

Os voos foram feitos entre os meses de outubro de 2016 e fevereiro de 2017. Foram executados 20 levantamentos em diferentes datas, horários e, consequentemente, números 


\section{VERIFICAÇÃO DA QUALIDADE DE ORTOMOSAICOS PRODUZIDOS A PARTIR DE IMAGENS OBTIDAS COM AERONAVE REMOTAMENTE PILOTADA SEM O USO DE PONTOS DE APOIO}

de satélites observáveis e condições meteorológicas (quadro 1).

Quadro 1 - Relação e características dos 20 voos

\begin{tabular}{|c|c|c|c|c|}
\hline N.o voo & Altura & Horário voo & Satélites & Condições metereólogicas \\
\hline 1 & $220 \mathrm{~m}$ & $09: 34$ & 17 & Nublado \\
\hline 2 & $220 \mathrm{~m}$ & $10: 15$ & 15 & Nublado \\
\hline 3 & $220 \mathrm{~m}$ & $11: 30$ & 17 & Sol entre nuvens \\
\hline 4 & $220 \mathrm{~m}$ & $14: 00$ & 16 & Sol entre nuvens \\
\hline 5 & $220 \mathrm{~m}$ & 09:30 & 16 & Nublado \\
\hline 6 & $220 \mathrm{~m}$ & $10: 43$ & 21 & Nublado \\
\hline 7 & $220 \mathrm{~m}$ & $11: 15$ & 20 & Nublado \\
\hline 8 & $220 \mathrm{~m}$ & $15: 25$ & 20 & Nublado \\
\hline 9 & $220 \mathrm{~m}$ & $15: 44$ & 19 & Nublado \\
\hline 10 & $220 \mathrm{~m}$ & 16:01 & 18 & Nublado \\
\hline 11 & $220 \mathrm{~m}$ & $11: 56$ & 19 & Sol \\
\hline 12 & $220 \mathrm{~m}$ & $12: 21$ & 16 & Sol \\
\hline 13 & $220 \mathrm{~m}$ & $14: 47$ & 18 & Sol \\
\hline 14 & $220 \mathrm{~m}$ & $16: 36$ & 19 & Nublado \\
\hline 15 & $220 \mathrm{~m}$ & 08:39 & 20 & Sol entre nuvens \\
\hline 16 & $220 \mathrm{~m}$ & $08: 56$ & 20 & Sol entre nuvens \\
\hline 17 & $220 \mathrm{~m}$ & 09:13 & 19 & Sol entre nuvens \\
\hline 18 & $220 \mathrm{~m}$ & 09:36 & 18 & Sol entre nuvens \\
\hline 19 & $220 \mathrm{~m}$ & $10: 34$ & 20 & Nublado \\
\hline 20 & $220 \mathrm{~m}$ & $10: 53$ & 19 & Nublado \\
\hline
\end{tabular}

Fonte: autores (2017).

O processamento dos dados foi executado no Software Agisoft PhotoScan. Foi aplicada a rotina de processamento sugerida pelo software (figura 2), o que envolve a orientação das fotos (align photos), identificação e densificação de pontos homólogos (Build dense cloud), geração dos modelos (Build mesh, texture, tiled model e DEM) e geração de ortomosaicos (Build orthomosaic) (Figura 2). As ortofotos foram geradas na resolução de 0,1 metros.
Para o processamento assumiu-se como desconhecidos os Parâmetros de Orientação Interior - POI da câmera (distância focal, coordenadas de ponto principal e coeficientes de distorção de lentes), sendo todos determinados em uma autocalibração. Conforme argumentado por Colomina e Molina (2014), testes realizados em softwares proprietários atestam a eficiência do procedimento. 
SOPCHAKI,C.H., PAZ,O.L.S., GRAÇA,N.L.Z.S., SAMPAIO,T.V.M.

\section{VERIFICAÇÃO DA QUALIDADE DE ORTOMOSAICOS PRODUZIDOS A PARTIR DE IMAGENS OBTIDAS COM AERONAVE REMOTAMENTE PILOTADA SEM O USO DE PONTOS DE APOIO}

\begin{tabular}{|c|c|c|}
\hline \multirow{2}{*}{ Align Photo } & General & $\begin{array}{l}\text { Accuracy: High } \\
\text { Pair preselection: Disabled }\end{array}$ \\
\hline & Advanced & $\begin{array}{l}\text { Key point limit: } 40,000 \text { - Tie point limit: } 4,000 \\
\text { Constrain features by mask: No } \\
\text { Adaptive camera model fitting: Yes }\end{array}$ \\
\hline \multirow[b]{2}{*}{ Build Dense Cloud } & General & Quality: Medium \\
\hline & Advanced & $\begin{array}{l}\text { Depth filtering: Aggressive } \\
\text { Reuse depth maps: No }\end{array}$ \\
\hline \multirow{2}{*}{ Build Mesh } & General & $\begin{array}{l}\text { Surface type: Arbitrary - Source data: Dense cloud } \\
\text { Face count: Medium - Custom face count: } 200,000\end{array}$ \\
\hline & Advanced & $\begin{array}{l}\text { Interpolation: Enabled (default) } \\
\text { Point classes: All }\end{array}$ \\
\hline \multirow[t]{2}{*}{ Build Texture } & General & $\begin{array}{l}\text { Mapping mode: Generic - Texture from: All Cameras } \\
\text { Blending mode: Mosaic } \\
\text { Texture size: } 4,096 \text { - Texture count: } 1\end{array}$ \\
\hline & Advanced & $\begin{array}{l}\text { Color correction: No } \\
\text { Hole filling: Yes }\end{array}$ \\
\hline Build Tiled Model & General & $\begin{array}{l}\text { Source data: Dense cloud } \\
\text { Pixel size: } 0 \text { - Tile size: } 256\end{array}$ \\
\hline Build DEM & General & $\begin{array}{l}\text { Source data: Dense cloud } \\
\text { Interpolation: Enabled (default) } \\
\text { Point class: All - Projection: Resolution (m/pix): } 0\end{array}$ \\
\hline Build Orthomosaic & General & $\begin{array}{l}\text { Resolution }(\mathrm{m}): 0 \\
\text { Surface: Mesh - Blending mode: Mosaic } \\
\text { Color Correction: No } \\
\text { Projection: - Use custom region: No }\end{array}$ \\
\hline
\end{tabular}

Figura 2 - Rotina de processamento dos dados coletados pela RPA no software Argisoft Photoscan. Fonte: autores (2017).

Os ortomosaicos tiveram sua qualidade planimétrica avaliada a partir de coordenadas de pontos de verificação levantados no terreno com GNSS ATX 900 Leica, que apresenta receptor de dupla frequência e permite o rastreio contínuo em 12 canais nas bandas L1 e L2. Estes pontos não foram utilizados para geração dos ortomosaicos. Demais especificações técnicas do equipamento estão disponíveis em Leica (2017). Os pontos foram levantados e processados na disciplina de Cartografia Geral, do Programa de Pós-Graduação em Ciências Geodésicas (UFPR), ministrada pela Profạ Drạ Luciene Stamato Delazari.
Foram selecionados 10 pontos (fig. 03) que pudessem ter sua posição identificada perfeitamente no terreno e nos ortomosaicos, a fim de calcular os erros. Cabe destacar que, conforme preconizam as normas e a literatura nacional e internacional (FGDC, 1998; MINNESOTA PLANNING, 1999; DSG, 2016a; DSG, 2016b) os pontos têm precisão pelo menos três vezes superior ao produto a ser avaliado e estão bem distribuídos por todos os quadrantes. 


\section{VERIFICAÇÃO DA QUALIDADE DE ORTOMOSAICOS PRODUZIDOS A PARTIR DE IMAGENS OBTIDAS COM AERONAVE REMOTAMENTE PILOTADA SEM O USO DE PONTOS DE APOIO}

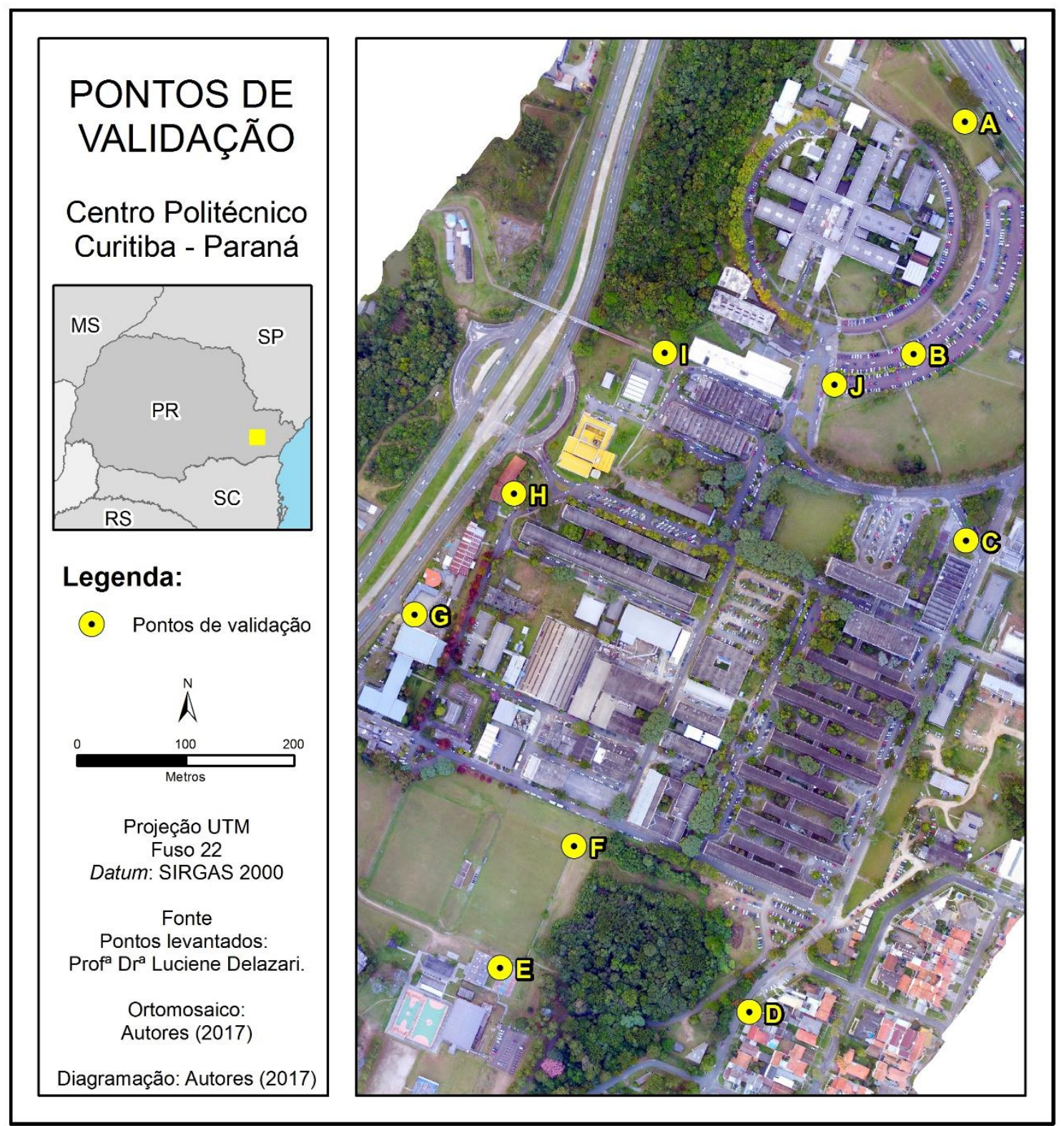

Figura 3 - Pontos amostrais para validação dos ortomosaicos. Fonte: autores (2017).

Conforme recomendação técnica da DSG (2016b) "o tamanho da amostra é determinado pelo processo de avaliação", sendo assim, a Teoria Exata da Amostragem (SPIEGEL, 1994) permite a adoção desta quantidade de pontos (10 pontos amostrais), assim como a quantidade de voos para validação (20 voos) (MERCHANT, 1982; GALO; CAMARGO, 1994; VIEIRA; GENRO; 2013).

As validações foram efetuadas a partir da análise de tendência por t-Student, e da precisão a partir do Erro Médio Quadrático (EMQ), conforme metodologia proposta por
FGDC (1998), MINNESOTA PLANNING (1999) e DSG (2016b). Foi calculado também o valor da acurácia absoluta para graus de confiança 90\% e para $95 \%$ a partir do EMQ (SOPCHAKI, 2016). A partir do cálculo de acurácia para 90\% e do EMQ, cada ortomosaico teve seu enquadramento efetuado junto ao PEC-PCD (DSG, 2016a) na maior escala possível para a Classe $A$.

A análise de tendência através da distribuição de t-Student, proposta por Merchant (1982) objetiva verificar se a média dos resíduos é estatisticamente nula, através do seguinte teste de hipótese: 


\section{VERIFICAÇÃO DA QUALIDADE DE ORTOMOSAICOS PRODUZIDOS A PARTIR DE IMAGENS OBTIDAS COM AERONAVE REMOTAMENTE PILOTADA SEM O USO DE PONTOS DE APOIO}

$$
\begin{aligned}
& H_{0}: \Delta \overline{x y}=0 \\
& H_{1}: \Delta \overline{x y} \neq 0
\end{aligned}
$$

Onde: $\Delta \overline{x y}$ equivale à média das discrepâncias das coordenadas das amostras.

$\mathrm{O} \mathrm{t}$ de Student amostral é calculado através da seguinte equação:

$$
t_{\text {calc }}=\frac{\Delta \overline{x y}}{\sigma} \sqrt{n}
$$

Onde: $\sigma$ equivale ao desvio padrão das discrepâncias das coordenadas das amostras e $n$ equivale ao tamanho da amostra validada.

O Desvio Padrão da amostra equivale à raiz quadrada positiva da variância, conforme a equação a seguir:

$$
S_{y}=\sqrt{S_{y}^{2}}
$$

Onde $S_{y}^{2}$ equivale à variância, a qual é calculada a partir da seguinte equação:

$$
S_{y}^{2}=\sum_{i=1}^{n} \frac{\left(\Delta x_{i}-\Delta \bar{x}\right)^{2}}{n-1}
$$

Onde $\Delta x_{i}$ refere-se à discrepância calculada em cada ponto e $\Delta \bar{x}^{-}$refere-se às médias das discrepâncias, obtidos, respectivamente, pelas seguintes equações:

$$
\begin{array}{r}
\Delta \boldsymbol{x}_{\boldsymbol{i}}=\boldsymbol{x}_{\text {ref } \boldsymbol{i}}-\boldsymbol{x}_{\text {test } \boldsymbol{i}}(\mathbf{6}) \\
\Delta \bar{x}=\frac{\sum_{i=1}^{n} \Delta x_{i}}{n} \\
\Delta y_{i}=y_{\text {ref } i}-y_{\text {test } i}(7) \\
\Delta \overline{\boldsymbol{y}}=\frac{\sum_{i=1}^{n} \Delta \boldsymbol{y}_{i}}{n}
\end{array}
$$

Onde: $x_{\text {ref } i}$ e $y_{\text {ref } i}$ referem-se-se às coordenadas tidas como referência para o teste (pontos de controle GNSS), $x_{\text {test } i}$ e $y_{\text {test } i}$ referem-se às coordenadas obtidas nos pontos homólogos identificados no ortomosaico

Se $\quad-t_{t a b} \leq t_{c a l c} \leq+t_{t a b}, \quad$ então aceita-se $H_{0}$. Caso contrário, rejeita-se $H_{0}$ e aceita-se $H_{1}$. $t_{\text {tab }}$ refere-se ao $t$-Student estatístico, de acordo com a probabilidade $(90 \%$ para o PEC-PCD) e o grau de liberdade (dado por $n-1)$. O t-Student estatístico pode ser obtido em tabelas de $t$ de Student ou diretamente em softwares de planilha eletrônica.

O Erro Médio Quadrático é calculado a partir da seguinte equação:

$$
E M Q_{x y}=\sqrt{\frac{\sum_{i=1}^{n}(x y)^{2}}{n}}(9)
$$

O cálculo da acurácia, a partir do EMQ, de acordo com a NSSDA (FGDC, 1998), para probabilidade de $95 \%$, é dado por:

Acurácia $(95 \%)=1,9600 \times E M Q(10)$

O cálculo da acurácia, a partir do EMQ, com probabilidade de 90\%, para fins de enquadramento junto ao PEC-PCD (DSG, 2016a) é dado por:

Acurácia $(90 \%)=1,6449 \times E M Q$

Além dos testes de acurácia posicional planimétrica absoluta, foram efetuadas também análises objetivando verificar a qualidade interna do modelo gerado. Para isto foram calculados os valores das distâncias cartográficas (UTM) entre as coordenadas de sete pares de pontos (Fig. 04) obtidos junto ao levantamento GNSS e as distâncias foram comparadas com as distâncias obtidas entre os pontos homólogos nos ortomosaicos, a partir das seguintes equações:

$$
\begin{array}{r}
\Delta x_{A-B}=x_{A}-x_{B} \\
\Delta y_{A-B}=y_{A}-y_{B} \\
\Delta x y_{A-B}=\sqrt{\left(\Delta x_{A-B}\right)^{2}+\left(\Delta y_{A-B}\right)^{2}} \\
{\text { Erro } x y_{A-B}}=\Delta x y_{A-B} \text { ref } i-\Delta x y_{A-B} \text { test } i
\end{array}
$$

Onde: $\Delta x_{A-B}$ refere-se à distância cartográfica entre os pontos $A$ e $B$ no eixo $X$; $\Delta y_{A-B}$ refere-se à distância cartográfica entre os pontos $\mathrm{A}$ e $\mathrm{B}$ no eixo $\mathrm{Y} ; \Delta x y_{A-B}$ refere-se à distância cartográfica resultante entre os pontos

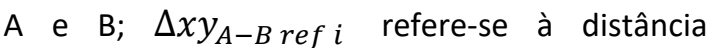
cartográfica entre os pontos $A$ e $B$, tidas como referência para $\mathrm{O}$ teste (pontos de controle

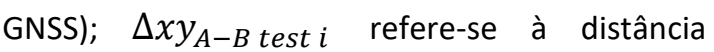
cartográfica entre os pontos A e B obtidas a partir do cálculo efetuado entre os pontos homólogos identificadas no ortomosaico; e $\operatorname{Erro} x y_{A-B}$ refere-se ao erro (em metros - distância 


\section{VERIFICAÇÃO DA QUALIDADE DE ORTOMOSAICOS PRODUZIDOS A PARTIR DE IMAGENS OBTIDAS COM AERONAVE REMOTAMENTE PILOTADA SEM O USO DE PONTOS DE APOIO}

cartográfica) calculado no ortomosaico entre os pontos A e B.

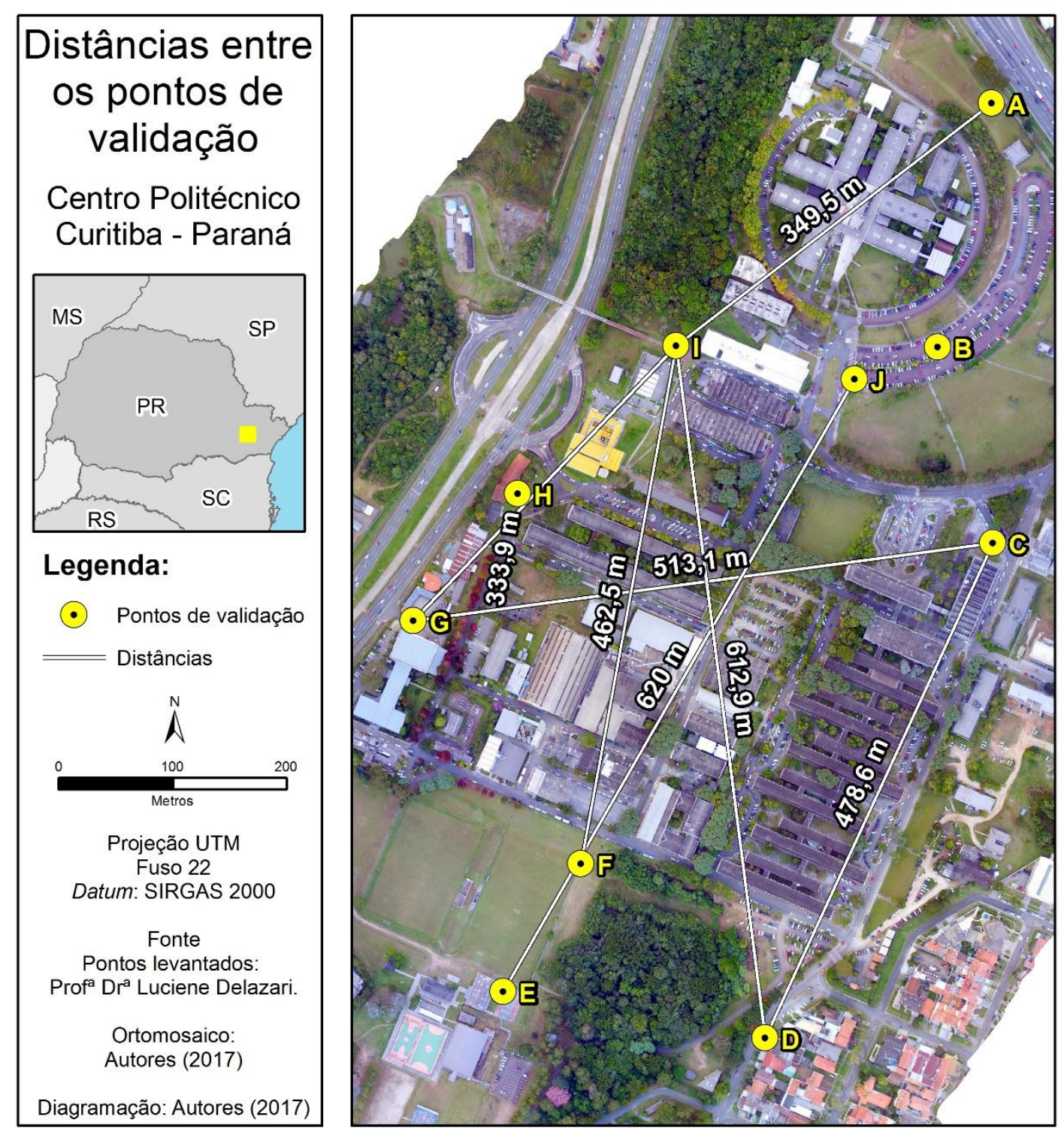

Figura 4 - Distância entre pontos amostrais. Fonte: autores (2017).

\section{RESULTADOS E DISCUSSÕES}

A figura 05, exposta a seguir, demonstra o resultado do cálculo do Root Mean Square Error - RMSE (Erro Médio Quadrático), os valores de acurácia absoluta para $95 \%$ e $90 \%$ dos pontos, o enquadramento junto ao PEC-PCD Classe A, na maior escala permitida para cada voo (com base nos valores de EMQ e acurácia), bem como os testes de t-Student efetuados, buscando averiguar se há alguma tendência de deslocamento. 


\section{VERIFICAÇÃO DA QUALIDADE DE ORTOMOSAICOS PRODUZIDOS A PARTIR DE IMAGENS OBTIDAS COM AERONAVE REMOTAMENTE PILOTADA SEM O USO DE PONTOS DE APOIO}

Ortomosaicos gerados a partir dos 20 voos realizados com Drone Phantom 3 no câmpus Centro Politécnico da UFPR

Acurácia planimétrica, enquadramento junto ao PEC-PCD e análise de tendência

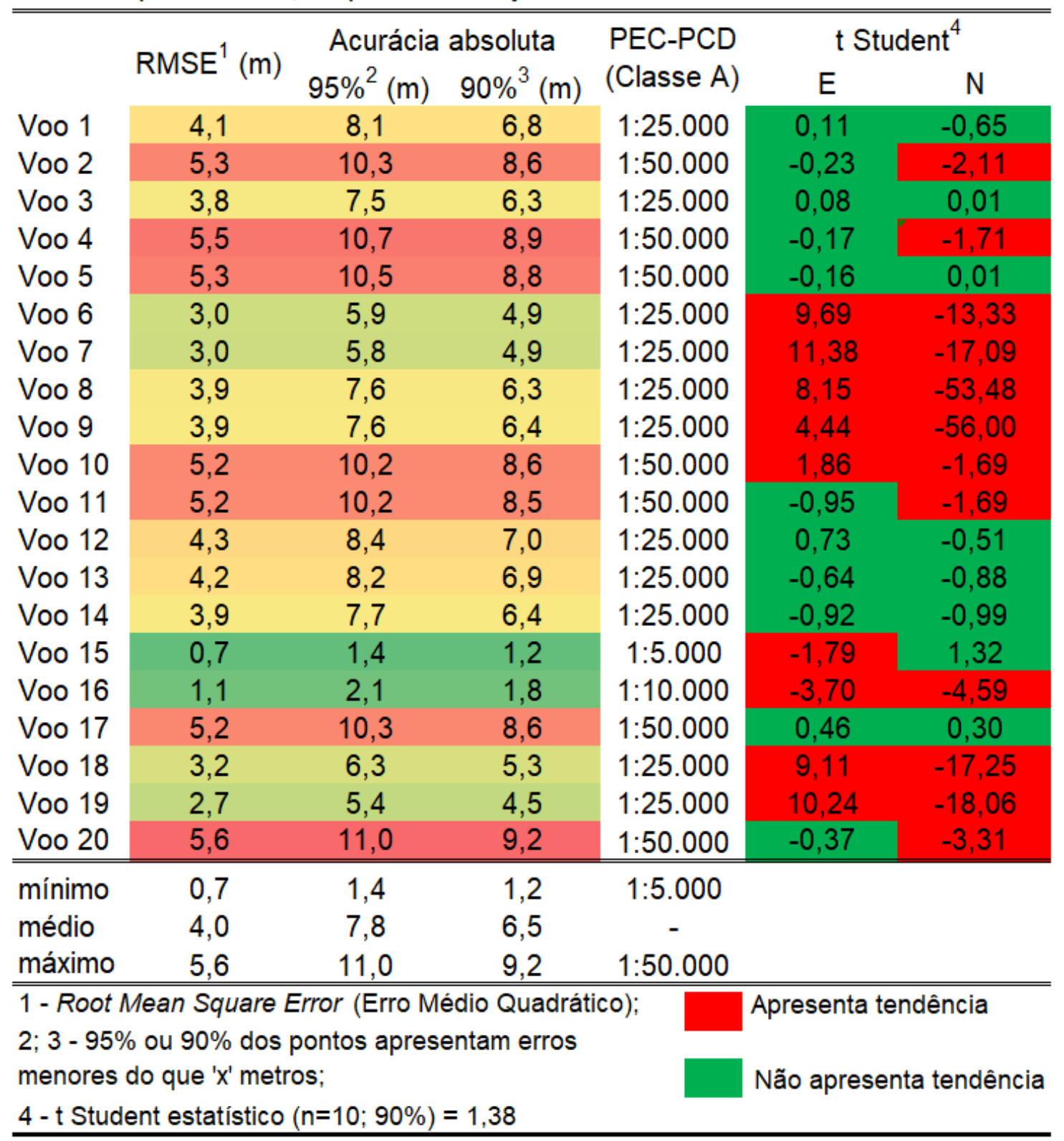

Figura 5 - Acurácia planimétrica dos 20 voos realizados no Centro Politécnico da UFPR Fonte: autores (2017).

A análise de tendência apresentou resultados compatíveis com o esperado para o tipo de produto gerado. Isto porque, há uma contribuição de erros intrínsecos ao posicionamento por GNSS. Conforme $\mathrm{Li}$ et al. (2015), Santerre et al. (2017) e Seeber (2003) há uma natural Diluição de Precisão - DOP em função da constelação de satélites rastreada.
Considerando que os voos foram realizados em uma janela de tempo inferior à 20min, e em datas e horários distintos, era esperado que a geometria das constelações satelitais observadas pudessem promover a ocorrência de tendência nas posições registradas para cada voo, quando analisado de forma isolada. Isto porque a geometria dos satélites, 


\section{VERIFICAÇÃO DA QUALIDADE DE ORTOMOSAICOS PRODUZIDOS A PARTIR DE IMAGENS OBTIDAS COM AERONAVE REMOTAMENTE PILOTADA SEM O USO DE PONTOS DE APOIO}

em períodos curto de tempo, provoca a introdução de erro sistemático.

Convém lembrar que a posição de cada imagem é derivada de uma trajetória calculada em tempo real (instantânea) a partir de dados inerciais e efemérides transmitidas. Portanto, não é possível afirmar que a tendência observada nos ortomosaicos é resultante exclusivamente da posição registrada nos metadados.

Infere-se, com base nos resultados explicitados na figura 05 , que o voo 15 apresentou menor valor de EMQ $(0,7 \mathrm{~m})$ e o voo 20 o maior valor $(5,6 \mathrm{~m})$. Assim, a acurácia absoluta para grau de confiança de $90 \%$ foi de 1,2 m para o voo 15 e 9,2 m para o voo 20 , o que permitiria que os ortomosaicos fossem enquadrados, respectivamente, nas escalas 1:5.000 e 1:50.000 do PEC-PCD Classe A (DSG, 2016a).

No tocante a acurácia absoluta para $95 \%$ dos pontos, o melhor resultado foi de 1,4 $\mathrm{m}$ (voo 15) e o pior resultado foi de $11,0 \mathrm{~m}$ (voo 20). 0 valor médio do RMSE dos 20 ortomosaicos foi de 4,0 m, a média da acurácia absoluta para $95 \%$ de confiança foi de $7,8 \mathrm{~m}$ e a média da acurácia absoluta para $90 \%$ de confiança foi de $6,5 \mathrm{~m}$.

Portanto, com base nessa amostragem de 20 voos realizados na mesma área, infere-se que os ortomosaicos produzidos com uso de RPAs-SPS (como o Phantom 3 Professional), associados ao uso de rotinas automazidadas (Agisoft Photoscan) e, sem uso de pontos de apoio apresentem enquadramento na escala 1:50.000 (podendo chegar a 1:5.000) conforme padrões do PEC-PCD Classe A (DSG, 2016a). Pode-se ainda inferir que, o voo deverá apresentar EMQ planimétrico médio de 4,0 m, estando, na melhor das hipóteses, com EMQ próximo a 0,7 m e, na pior das hipóteses, com EMQ próximo a 5,6 m.

Quanto à análise de tendência, 65\% dos voos apresentaram deslocamento sistemático, conforme resultados obtidos a partir dos testes de t-Student. Quatro ortomosaicos apresentaram tendência de deslocamento em somente um eixo (voos 2, 4, 15 e 20) e os demais (voos 6, 7, 8, 9, $10,16,18$ e 19) apresentaram tendência de deslocamento em ambos os eixos. Neste caso, seria pertinente avaliar a possibilidade de corrigir a tendência e, se a remoção da mesma pode resultar em melhoria na qualidade posicional.

A Figura 06 apresenta os resultados da análise da qualidade interna do modelo. Buscouse, a partir desta análise, verificar se havia um padrão predominante de propagação de erros (radial, perimetral, longitudinal, latitudinal). 
VERIFICAÇÃO DA QUALIDADE DE ORTOMOSAICOS PRODUZIDOS A PARTIR DE IMAGENS OBTIDAS COM AERONAVE REMOTAMENTE PILOTADA SEM O USO DE PONTOS DE APOIO

\begin{tabular}{|c|c|c|c|c|c|c|c|c|c|c|c|c|c|}
\hline \multicolumn{14}{|c|}{$\begin{array}{l}\text { Distância cartográfica entre os pares de pontos GNSS e erros obtidos nas } \\
\text { medidas efetuadas entre os pontos homólogos nos Ortomosaicos }\end{array}$} \\
\hline Pontos & GNSS $^{1}$ & Orto $^{2}$ & \multicolumn{2}{|c|}{$\begin{array}{l}\text { Erro } \\
)^{3}(\%)^{4}\end{array}$} & Orto $^{2}$ & \multicolumn{2}{|c|}{$\begin{array}{l}\text { Erro } \\
)^{3}(\%)^{4}\end{array}$} & Orto $^{2}$ & \multicolumn{2}{|c|}{$\begin{array}{l}\text { Erro } \\
1)^{3}(\%)^{4}\end{array}$} & Orto $^{2}$ & $(m)^{3}$ & $(\%)^{4}$ \\
\hline & & & Voo 1 & & & Voo 2 & & & Voo 3 & & & Voo 4 & \\
\hline C. $-\mathrm{G}$ & 513,1 & 506,2 & 6,9 & $1,35 \%$ & 505,2 & 7,9 & $1,54 \%$ & 506,5 & 6,6 & $1,28 \%$ & 504,6 & 8,5 & $1,65 \%$ \\
\hline$E-J$ & 620,0 & 611,9 & & $31 \%$ & 610,7 & & $1,51 \%$ & 612,3 & 7,8 & $1,25 \%$ & 610,2 & 9,9 & $1,59 \%$ \\
\hline A - I & 349,5 & 345,2 & 4 & $23 \%$ & 345,0 & 4,4 & $1,26 \%$ & 345,6 & 3,9 & $1,12 \%$ & 344,2 & 5,3 & $1,51 \%$ \\
\hline G-I & 333,9 & 329,4 & 4 & 6 & 328,6 & & $58 \%$ & 329,4 & 4,5 & $34 \%$ & 328,4 & 5,5 & $1,65 \%$ \\
\hline D - C & 478,6 & 472 & 6 & $\%$ & 471,5 & & $49 \%$ & 472 & 5,9 & $1,24 \%$ & 471,2 & 7,5 &, $56 \%$ \\
\hline $\mathrm{I}-\mathrm{F}$ & 462,5 & 456 & 6,0 & $\%$ & 455,4 & & $5 \%$ & & 5,8 & $1,26 \%$ & 454,9 & 7,6 & $1,65 \%$ \\
\hline \multirow[t]{2}{*}{ I-D } & 612,9 & 605,0 & 8,0 & $1,30 \%$ & 603,6 & 9,4 & $1,53 \%$ & 605,2 & 7,8 & $1,27 \%$ & 603,0 & 10,0 & $1,63 \%$ \\
\hline & & \multicolumn{3}{|c|}{ Voo 5} & \multicolumn{3}{|c|}{ Voo 6} & \multicolumn{3}{|c|}{ Voo 7} & \multicolumn{3}{|c|}{ Voo 8} \\
\hline C - G & 513,1 & 505,4 & 7,7 & $1,50 \%$ & 514,0 & $-0,9$ & $-0,18 \%$ & 513,8 & $-0,7$ & $-0,14 \%$ & 512,4 & 0,7 & $0,13 \%$ \\
\hline$E-J$ & 620,0 & 610,0 & 10,1 & 1,62 & 621,6 & & $-0,25 \%$ & 621 & $-1,4$ & $-0,22 \%$ & 620,3 & $-0,3$ & $-0,05 \%$ \\
\hline A - I & 349,5 & 344,2 & 5,3 & $1,50 \%$ & 350,1 & -0 , & $-0,18 \%$ & 349 & $-0,2$ & $-0,04 \%$ & 349,1 & 0,3 & $0,09 \%$ \\
\hline G - I & 333,9 & 328,6 & 5,3 & $1,59 \%$ & 335,0 & $-1,1$ & $-0,33 \%$ & 335 & $-1,3$ & $-0,40 \%$ & 333,8 & 0,0 & $0,01 \%$ \\
\hline D - C & 478,6 & 471, & 7,6 & $1,58 \%$ & 479,7 & $-1,0$ & $-0,22 \%$ & 479,3 & $-0,6$ & $-0,13 \%$ & 478,4 & 0,3 & $0,05 \%$ \\
\hline $\mathrm{I}-\mathrm{F}$ & 462,5 & 454,9 & 7,6 & $1,64 \%$ & 463,6 & $-1,1$ & $-0,24 \%$ & 463,4 & $-0,9$ & $-0,19 \%$ & 462,3 & 0,2 & $0,04 \%$ \\
\hline \multirow[t]{2}{*}{ I-D } & 612,9 & 602,9 & 10,0 & $1,63 \%$ & 613,9 & $-0,9$ & $-0,15 \%$ & 613,4 & $-0,4$ & $-0,07 \%$ & 612,1 & 0,9 & $0,15 \%$ \\
\hline & & \multicolumn{3}{|c|}{ Voo 9} & \multicolumn{3}{|c|}{ Voo 10} & \multicolumn{3}{|c|}{ Voo 11} & \multicolumn{3}{|c|}{ Voo 12} \\
\hline C - G & 513,1 & 512,4 & 0,7 & $0,14 \%$ & 506,0 & 7,0 & $1,37 \%$ & 505,1 & 8,0 & $1,55 \%$ & 505,9 & 7,2 & $1,41 \%$ \\
\hline$E-J$ & 620,0 & 620 & & & 611,8 & & & & & & 612 & 8 & \\
\hline A - I & 349,5 & 349 & & & 345 & 3 & & & & & 34 & 6 & $1 \%$ \\
\hline G - I & 333,9 & & & & & & & & & & & 8 & $4 \%$ \\
\hline D - C & 478,6 & 4 & & & 72,3 & & & & & & 472,2 & 6,4 & $5 \%$ \\
\hline $\mathrm{I}-\mathrm{F}$ & 462,5 & 462 & 0,2 & & 456,2 & 6,3 & $1,37 \%$ & & & & 456,1 & 6,4 & $1,39 \%$ \\
\hline I-D & 612,9 & 612,2 & 0,8 & $0,12 \%$ & 604,6 & 8,3 & $1,36 \%$ & 603,6 & 9,4 & $1,53 \%$ & 604,6 & 8,3 & $1,36 \%$ \\
\hline & & & Voo 13 & & & Voo & & & Voo 15 & & & Voo 16 & \\
\hline C - G & 513,1 & 506,0 & 7,1 & $1,38 \%$ & 506,9 & 6,2 & $1,20 \%$ & 513,8 & $-0,7$ & $-0,13 \%$ & 512,0 & 1,1 & $0,21 \%$ \\
\hline$E-J$ & 620,0 & 612,5 & 7,5 & $1,22 \%$ & 612,9 & 7,2 & $1,15 \%$ & 621,7 & $-1,6$ & $-0,26 \%$ & 619,6 & 0,5 & $8 \%$ \\
\hline A - I & 349,5 & 345,5 & 4,0 & $1,14 \%$ & 345,6 & 3,8 & $1,09 \%$ & 350,3 & $-0,8$ & $-0,24 \%$ & 348,9 & 0,6 & $0,16 \%$ \\
\hline G - I & 333,9 & 329,2 & 4,7 & $1,39 \%$ & 329,9 & 4,0 & $1,19 \%$ & 334,4 & $-0,5$ & $-0,16 \%$ & 333,5 & 0,3 & $0,10 \%$ \\
\hline D - C & 478,6 & 471,9 & 6 , & $1,40 \%$ & 472,7 & 5,9 & $1,24 \%$ & 479,6 & $-0,9$ & $-0,19 \%$ & 478,0 & 7 & $0,14 \%$ \\
\hline $\mathrm{I}-\mathrm{F}$ & 462,5 & 456 & & & & 5 , & & 463,4 & $-0,8$ & $8 \%$ & 461,9 & 0,7 & $5 \%$ \\
\hline I-D & 612,9 & 604,4 & 8,5 & $1,39 \%$ & 605,4 & 7,6 & $1,23 \%$ & 613,7 & $-0,8$ & $-0,13 \%$ & 611,7 & 1,3 & $0,20 \%$ \\
\hline & & & & & & & & & & & & & \\
\hline C - G & 513,1 & 504,3 & & & 513,6 & $-0,5$ & & 512,2 & 0,9 & & 505,5 & 7,6 & \\
\hline$E-J$ & 620,0 & 609,8 & 10,3 & & 621,8 & $-1,7$ & $-0,28 \%$ & 619,6 & 0,5 & & 611,9 & 8,2 & $2 \%$ \\
\hline A - I & 349,5 & 344,0 & 5,5 & 1,58 & 349,8 & $-0,3$ & $-0,10 \%$ & 349,1 & 0,3 & & 344,7 & 4,8 & $1,36 \%$ \\
\hline G - I & 333,9 & 327,9 & 5,9 & $1,78 \%$ & 334,7 & $-0,8$ & $-0,24 \%$ & 333,6 & 0,3 & $0,09 \%$ & 328,9 & 4,9 & $1,48 \%$ \\
\hline D - C & 478,6 & 470,8 & 7,8 & $1,64 \%$ & 479,0 & $-0,3$ & $-0,07 \%$ & 478,3 & 0,3 & $0,07 \%$ & 472,0 & 6,7 & $1,39 \%$ \\
\hline $\mathrm{I}-\mathrm{F}$ & 462,5 & 454,5 & 8,0 & $1,73 \%$ & 463,6 & $-1,1$ & $-0,24 \%$ & 462,0 & 0,6 & $0,12 \%$ & 456,0 & 6,6 & $1,42 \%$ \\
\hline I-D & 612,9 & 602,5 & 10,4 & $1,70 \%$ & 613,4 & $-0,5$ & $-0,08 \%$ & 612,0 & 1,0 & $0,16 \%$ & 604,3 & 8,6 & $1,41 \%$ \\
\hline
\end{tabular}

Figura 5 - Erros internos dos 20 ortomosaicos. Fonte: autores (2017).

Com o apoio da figura 05, que apresenta a distribuição dos pontos, é possível concluir que não há um padrão de distribuição dos erros, mas que os mesmos se distribuem uniformemente nos ortomosaicos, conforme pode se observar nos resultados dos erros apresentados em percentagem.

Tomando como exemplo o voo 1, podese inferir que os erros variam de 4,3 m (distância cartográfica entre os pontos $A-1$ ) até $8,2 \mathrm{~m}$ 


\section{VERIFICAÇÃO DA QUALIDADE DE ORTOMOSAICOS PRODUZIDOS A PARTIR DE IMAGENS OBTIDAS COM AERONAVE REMOTAMENTE PILOTADA SEM O USO DE PONTOS DE APOIO}

(distância cartográfica entre os pontos E - J). Vistos em percentagem, os valores dos erros são de aproximadamente $1,3 \%$ do valor da dimensão medida, independente do padrão geométrico e da localização dos pontos no ortomosaico, ou seja, no geral, serão maiores os erros, quanto maiores forem as distâncias.

Alguns voos apresentaram padrão de acurácia posicional interna (no que se refere à planimetria) superior ao padrão de posicionamento planimétrico calculado anteriormente. Embora os resultados de acurácia planimétrica tenham demonstrado que o melhor voo foi o de $n$ ㅇ 15 e o pior voo foi o de $n$ ㅇ 20 , o mesmo não se repetiu com os resultados da qualidade interna.

Os voos que obtiveram os menores percentuais de erros nas distâncias cartográficas medidas diretamente nos ortomosaicos foram os voos 8 e 9, ambos com média dos erros de 0,06\%. Tais voos obtiveram EMQ de $3,9 \mathrm{~m}$, portanto, embora enquadrados na escala 1:25.000 do PECPCD, no que diz respeito ao processo de obtenção de distâncias planimétricas, os erros observados são inferiores aos esperados para esta escala.

Os voos que apresentaram os maiores percentuais de erros de distância cartográfica planimétrica entre os pontos foram os voos 5,4 e 17, com valores médios dos erros respectivamente de, $1,58 \%, 1,60 \%$ e $1,69 \%$.

Conclui-se, portanto, com base na análise dos resultados dos 20 voos realizados, que, ao utilizar uma RPA (Drone Phantom 3 Professional), atuando no modo de processamento sem controle de campo, apoiando-se tão somente nos dados de posição registrados nos metadados de cada imagem e com uma autocalibração da câmera, espera-se que o EMQ esteja abaixo de 5,6 $\mathrm{m}$ e que o valor em percentagem dos erros entre as distâncias internas seja inferior a 1,69\% da distância cartográfica de referência (verdade de campo).

O objetivo da pesquisa foi o de informar aos pesquisadores que demandam dados e, que desejam utilizar RPA sem pontos de apoio, qual a estimativa de erro associada ao posicionamento do ortomosaico e, as medidas obtidas diretamente sobre estes produtos, como exemplo, a mensuração de uma voçoroca.
Neste caso, é possível afirmar que no pior cenário observado, o enquadramento do ortomosaico em relação a sua qualidade posicional será 1:50.000 conforme PEC-PCD Classe A, 1:25.000 (Classes B e C) e 1:10.000 (Classe D), ainda que, as dimensões obtidas sobre este tipo de produto apresentam erro não superior a $1,69 \%$ da dimensão medida.

\section{CONSIDERAÇÕES FINAIS}

A pesquisa aborda a qualidade dos ortomosaicos produzidos com RPAs-SPS, uso de rotinas automatizadas e sem pontos de apoio. $\mathrm{O}$ uso de pontos de apoio, alteração de rotinas e softwares e, do equipamento podem produzir valores diferentes dos daqui observados.

Os valores demonstram que o uso de rotinas automatizadas de softwares especialistas possibilita a geração de ortomosaicos na escala 1:50.000 ou melhores (Classe A do PEC-PCD), sem o emprego de pontos de apoio. Demonstram também que as dimensões obtidas sobre os ortomosaicos não apresentaram erros superiores a $1,69 \%$, podendo chegar a $0,06 \%$.

Cabe destacar ainda que a pesquisa demonstrou que não há um padrão predominante de propagação de erros (radial, perimetral, longitudinal, latitudinal), pois os erros independem do padrão geométrico e da localização dos pontos no ortomosaico, ou seja, serão maiores os erros, quanto maiores forem as distâncias.

A pesquisa utilizou ortomosaicos construídos a partir de um único voo, ou seja, os valores observados não se aplicam a ortomosaicos construídos a partir do uso de dados de múltiplos voos.

\section{AGRADECIMENTOS}

Aos discentes da disciplina de Cartografia Geral, do Programa de PósGraduação em Ciências Geodésicas (UFPR) e à Profa Dra Luciene Stamato Delazari, por terem, gentilmente, cedido os dados do levantamento elaborado com GNSS no câmpus Centro Politécnico.

\section{REFERÊNCIAS}

ANAC - AGÊNCIA NACIONAL DE AVIAÇÃO CIVIL. RPAS - Sistemas de Aeronaves Remotamente 


\section{VERIFICAÇÃO DA QUALIDADE DE ORTOMOSAICOS PRODUZIDOS A PARTIR DE IMAGENS OBTIDAS COM AERONAVE REMOTAMENTE PILOTADA SEM O USO DE PONTOS DE APOIO}

Pilotadas. ANAC, 2015. Disponível em <https://goo.gl/MTjt67>. Acesso em 08 out 2017.

BRAZ, A. M.; BORGES, J. P. dos S.; BERNARDES, D. C. da S.; TEREZAN, L. H. Análise da aplicação de VANT na atualização de cadastro florestal com uso de pontos de controle. In: XVII Simpósio Brasileiro de Sensoriamento Remoto - SBSR, João Pessoa-PB, Brasil, INPE, 1, Anais...2015.

BUFFON, E. A. M.; PAZ, O. L. de S. da; SAMPAIO, T. V. M. Uso de Veículo Aéreo Não Tripulado (Vant) Para Mapeamento das Vulnerabilidades à Inundação Urbana: Referenciais e Bases de Aplicação. Revista do Departamento de Geografia, n. spe, p. 180, 2017.

CAI, C.; GAO, Y. Precise Point Positioning Using Combined GPS and GLONASS Observations. Journal of Global Positioning Systems, v. 6, n. 1, p. 13-22, 2007.

CÂNDIDO, A. K. A. A.; DA SILVA, N. M.; FILHO, A. C. P. Imagens de alta resolução espacial de Veículos Aéreos Não Tripulados (VANT) no planejamento do uso e ocupação do solo. Anuario do Instituto de Geociencias, v. 38, n. 1, p. 147-156, 2015.

COLOMINA, I.; MOLINA, P. Unmanned aerial systems for photogrammetry and remote sensing: A review. Journal of Photogrammetry and Remote Sensing, 2014.

DA SILVA, C. A.; DUARTE, C. R.; SOUTO, M. V. S.; SABADIA, J. A. B. Utilização de VANT para geração de ortomosaicos e aplicação do Padrão de Exatidão Cartográfica (PEC). XVII Simpósio Brasileiro de Sensoriamento Remoto - SBSR, Jo\{ã\}o Pessoa-PB, Brasil, INPE, n. 1, p. 6381-6388, 2015.

DECEA - DEPARTAMENTO DE CONTROLE DO ESPAÇO AÉREO. Portaria DECEA № 282/DGCEA, de 22 de dezembro de 2016. Aprova a reedição da ICA 100-40, que trata dos "Sistemas de Aeronaves Remotamente Pilotadas e o Acesso ao Espaço Aéreo Brasileiro". Publicado no BCA, Brasília, DF, 02 fev 2017.

DEPARTMENT OF DEFENSE, U. Global Positioning System Standard Positioning Service. Department Of Defense, U.S.AWashingtonDepartment of Defense, USA, , 2008.

DJ. Phantom 3 Professional Downloads. Disponível em < https://goo.gl/PAuP91>. Acesso em 08 ago 2017.
DSG - DIRETORIA DO SERVIÇO GEOGRÁFICO. Especificação técnica para a aquisição de dados geoespaciais vetoriais (ET-ADGV). 2a ed., Versão 1.1, $2016 a$.

DSG - DIRETORIA DO SERVIÇO GEOGRÁFICO. Especificação técnica para controle de qualidade de dados geoespaciais (ET-CQDG). 1ạ ed., 2016b.

EVERAERTS, J. The use of unmanned aerial vehicles (UAVs) for remote sensing and mapping. The International Archives of the Photogrammetry, Remote Sensing and Spatial Information Sciences, v. 37, n. 2008, p. 11871192, 2008.

FGDC. National Standard for Spatial Data Accuracy. Federal Geographic Data Committee, 1998. Disponível em: <http://goo.gl/SLX7i6> Acesso em 20 ago 2014.

GALO, M.; CAMARGO, P. de O.; O uso do GPS no controle de qualidade de cartas. In.: COBRAC 1994, 10 Congresso Brasileiro de Cadastro Técnico Multifinalitário. Tomo II, p. 41-48, Florianópolis - SC, 1994.

GUJJAR, P.; RAO, P.; RAO, P. S.; DEVI, G. L. The MIR Flickr Retrieval Evaluation Proposal Based on User Tags and Textual Passwords. International Journal of Advanced Engineering, Management and Science, n. 4, p. 343-349, 2017.

HARDIN, P. J.; JENSEN, R. R. Small-scale unmanned aerial vehicles in environmental remote sensing: Challenges and opportunities. GIScience \& Remote Sensing, v. 48, n. 1, p. 99111, 2011.

LEICA. Leica GPS900 Especificaciones Técnicas. Disponível em: <https://goo.gl/o4k8Mk>. Acesso em: 13 Nov 2017.

LI, X.; ZHANG, X.; REN, X.; FRITSCHE, M.; WICKERT, J.; SCHUH, H. Precise positioning with current multi-constellation Global Navigation Satellite Systems: GPS, GLONASS, Galileo and BeiDou. Scientific Reports, v. 5, p. 8328, fev. 2015.

LONGHITANO, G. A. VANTS para sensoriamento remoto: aplicabilidade na avaliação e monitoramento de impactos ambientais causados por acidentes com cargas perigosas. 2010. Tese de Doutorado. Universidade de São Paulo, 2010.

LUCIEER, A.; JONG, S. M. de; TURNER, D. Mapping landslide displacements using Structure from 


\section{VERIFICAÇÃO DA QUALIDADE DE ORTOMOSAICOS PRODUZIDOS A PARTIR DE IMAGENS OBTIDAS COM AERONAVE REMOTAMENTE PILOTADA SEM O USO DE PONTOS DE APOIO}

Motion (SFM) and image correlation of multitemporal UAV photography. Progress in Physical Geography, v. 38, n. 1, p. 97-116, 2014.

MARCHETTI, D. A. B.; GARCIA, G. J. Princípios de fotogrametria e fotointerpretação. [s.l.] Nobel, 1989.

MERCHANT, D.C. Spatial accuracy standards for large scale line maps. AMERICAN CONGRESS ON SURVEYING AND MAPPING. USA - Denver/CO, 1982.

MINNESOTA PLANNING. Positional Accuracy Handbook. Using the National Standard for Spatial data Accuracy to measure and report geographic data quality. Minnesota Planning, St. Paul, MN, 1999.

MOHAMMAD, S.; KHAN, N.; MOHD, J.; QADRI, L. The Understanding of Exchangeable Image File ( Exif ) Metadata of Images: Towards Disseminating the Awareness to the Society. In: 3th International Conference on Information Technology \& Society, Anais...2017.

NEX, F.; REMONDINO, F. UAV for 3D mapping applications: A review. Applied Geomatics, 2014.

PERIN, G.; GERKE, T.; LACERDA, V. S.; ZUBEK DA ROSA, J.; CAIRES, E. F.; GUIMARÃES, A. M. Análise de Acurácia de Georrefereciamento de Mosaicos de Imagens Obtidas por RPA. Anais do EATI Encontro Anual de Tecnologia da Informação e STIN - Simpósio de Tecnologia da Informação da Região Noroeste do RS 193, p. 193-199, 2016.

PRIMICERIO, J.; DI GENNARO, S. F.; FIORILLO, E.; GENESIO, L.; LUGATO, E.; MATESE, A.; VACCARI, F. P. A flexible unmanned aerial vehicle for precision agriculture. Precision Agriculture, v. 13, n. 4, p. 517-523, 2012.

RANGO, A.; LALIBERTE, A.; HERRICK, J. E.; WINTERS, C.; HAVSTAD, K.; STEELE, C.; BROWNING, D. Unmanned aerial vehicle-based remote sensing for rangeland assessment, monitoring, and management. Journal of Applied Remote Sensing, v. 3, n. 1, p. 33542, 2009.

SANTERRE, R.; GEIGER, A.; BANVILLE, S. Geometry of GPS dilution of precision: revisited. GPS Solutions, v. 21, n. 4, p. 1747-1763, 2017.
SEEBER, G. Satellite Geodesy: Foundations, Methods, and Applications. [s.I.] De Gruyter, 2003.

SOLOMON, D.; LEHMANN, J.; MAMO, T.; FRITZSCHE, F.; ZECH, W. Phosphorus forms and dynamics as influenced by land use changes in the sub-humid Ethiopian highlands. Geoderma, v. 105, n. 1-2, p. 21-48, 2002.

SOPCHAKI, C. H. Influência do $\mathrm{N}$ amostral e das características do relevo na qualidade de modelos digitais do terreno. Tese (doutorado) Universidade Federal do Paraná, Setor de Ciências da Terra, Programa de Pós-Graduação em Geografia. 161 f. Curitiba, 2016.

SOUSA, A.; SOUSA, J. J.; COSTA, J. DETEÇÃO DE OBJETOS A PARTIR DE IMAGENS OBTIDAS POR VANT. (U. N. de Lisboa, Ed.) In: SEMINÁRIO INTERNACIONAL UAV, Lisboa. Anais... Lisboa: Universidade Nova de Lisboa, 2016.

SOUSA, H. L. de. Sensoriamento Remoto com VANTs: uma nova possibilidade para a aquisição de geoinformações. Revista Brasileira de Geomática, p. 326-342, 2017.

SPIEGEL, M.R. Estatística: 383 Problemas Resolvidos, 416 Problemas Suplementares. Tradução e revisão técnica: Pedro Consentino. 3ạ edição. São Paulo: Pearson Education do Brasil, 1994.

SUMALAN, A. L.; POPESCU, D. A. N.; ICHIM, L. Flooded areas detection based on LBP from UAV images. Recent Advances on Systems, Signals, Control, Communications and Computers, p. 186-191, 2015.

TORRADO, J. O. E. Ortomosaicos y modelos digitales de elevación generados a partir de imágenes tomadas con sistemas UAV. Tecnura, v. 20, n. 50, p. 119-140, 2016.

VIEIRA, H. B. G.; GENRO, R. S. Estimativa da acurácia posicional de documentos cartográficos na Petrobras a partir do erro máximo provável inferido do erro médio quadrático e da respectiva variância propagada. XVI Simpósio Brasileiro de Sensoriamento Remoto - SBSR, Foz do Iguaçu, PR, 2013.

ZANETTI, J. Influência do número e distribuição de pontos de controle em ortofotos geradas a partir de um levantamento por VANT. 2017. UFV, 2017 